\section{Orbital Hemorrhage as a Complication of Gastroscopy}

Diagnostic gastroscopy is a safe procedure with a complication rate of below $0.1 \%$. These complications are mostly cardiopulmonary and, sometimes, rare instances of perforation, bleeding and infection $(1,2)$. We report the first case of an orbital hemorrhage secondary to gastroscopy.

A 35-year-old woman, who stated that she did not take regular medication although she had suffered from left-sided migraine for many years, had a prior neurological examination, including an enhanced computed tomography (CT) scan, with normal findings.

At a previous gastroscopy an insufficient cardia had been diagnosed. Because of suspected hematemesis, another gastroscopy with local pharyngeal anesthesia was performed 3 years later by a senior endoscopist using an Olympus videogastroscope. Apart from moderate attacks of retching, nothing abnormal was registered, but immediately after the examination the patient complained of pain behind the left eye. Despite absence of any clinical signs of damage to the eye, the patient was sent for ophthalmological examination. During the next hour diplopia developed and there was ecchymosis of the eyelids on the left side. The left globe was proptosed $6 \mathrm{~mm}$ and infraplaced with restricted motility (Figure 1). Visual acuity, intraocular pressure, pupillary reactions and ophthalmoscopic appearance were normal bilaterally. A CT scan demonstrated a hematoma causing displacement of the eye (Figure 2), with restitution 3 and 7 weeks later. Gadolinium-enhanced magnetic resonance imaging (MRI) angiography 5 months later revealed no vascular anomaly and the patient is still well 4 years later.

Spontaneous orbital hemorrhage is uncommon and has been described in connection with venous anomalies and with advanced atherosclerosis, hypertension, anemia and hemophilia being considered as predisposing conditions (3). Straining is another associated factor (3-5), probably causative in our case. As orbital veins have no valves, an unobstructed venous pressure, secondary to an increased intrathoracic pressure, is freely propagated to the orbital veins $(3,5)$. In our patient the intubationrelated, transitory increase in thoracic pressure, probably exaggerated by the attacks of retching, may have caused the orbital hemorrhage.

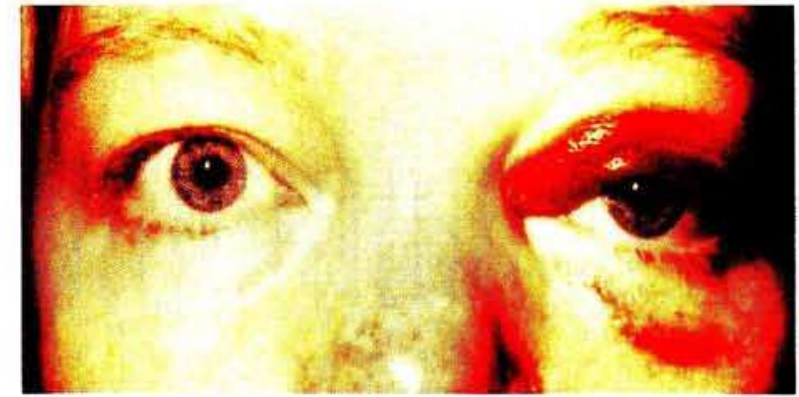

Figure 1: Ecchymosis of the eyelids on the left side due to expansion of an orbital hematoma. The left globe is proptosed and infraplaced. The nose has coexisting rosacea.

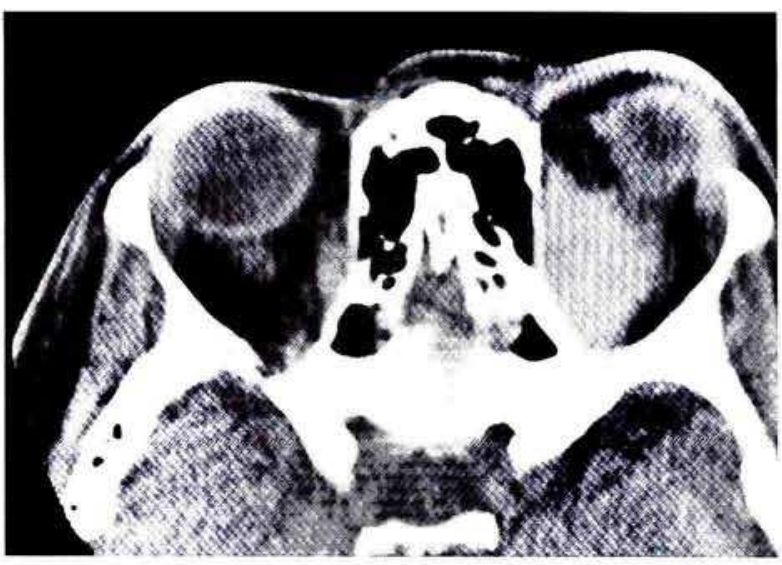

Figure 2: Non-contrast orbital computed tomography scan, demonstrating leftsided proptosis and an extraconal superior hematoma measuring $2 \times 2 \times 1 \mathrm{~cm}$.

\section{E. Tóth', U. Havelius ${ }^{2}$, F-T. Fork}

${ }^{1}$ Endoscopy Unit, Dept. of Diagnostic Radiology, Malmö University Hospital, University of Lund, Malmö, Sweden

${ }^{2}$ Dept. of Ophthalmology,

Malmö University Hospital,

University of Lund, Malmö, Sweden

\section{References}

1. Hart R, Classen M. Complication of diagnostic gastrointestinal endoscopy. Endoscopy 1990; 22: 229-33.

2. Eimiller A. Complication in endoscopy. Endoscopy 1992; 24: 176-84.

3. Krohel GB, Wright JE. Orbital hemorrhage. Am J Ophthalmol 1979; 88: 254-8.
4. Law FW. Spontaneous orbital hemorrhage. $\mathrm{Br} \mathrm{J}$ Ophthalmol 1971; 55: $556-8$.

5. Reese $A B$. Tumors of the eye. New York: Harper and Row, 1963: 547.

Corresponding Author

E. Tóth, M.D.

Endoscopy Unit

Dept. of Diagnostic Radiology

Malmö University Hospital

University of Lund

20502 Malmö

Sweden

Fax: + 46-40-969977 